\title{
La formación del profesorado ante la diversidad de creencias religiosas en el máster de formación de profesorado de enseñanza secundaria. Percepción docente
}

\author{
Teacher's training regarding diversity of religion beliefs. \\ Teachers' perception.
}

ISSN 1510-2432 - ISSN 1688-9304 (en línea) - DOI: https://doi.org/10.18861/cied.2018.9.1.2821

María Ángeles Hernández Prados

Doctora y Licenciada en Pedagogía, Universidad de Murcia, España. Profesora e Investigadora en Educación para la ciudadanía y mejora de la convivencia escolar, educación en valores en el contexto familiar y perspectiva ético-moral de la sociedad de la información, y la relación entre familia y centros escolares.

David Ibáñez Bordallo

Doctor e Investigador (Cand.) en Educación sobre políticas, prácticas y evaluación en contextos fomativos y socioeducativos. Máster en Orientación Educativa, Universidad de Murcia. Maestro de Educación Primaria. Expecialista en Historia y Filosofía de las religiones, Fundación Xavier Zubiri, España.

Fecha de recibido: 04/07/2017

Fecha de aceptado: 22/03/2018

\section{Resumen:}

Nuestro contexto actual cuenta con un panorama rico en diversidad de creencias que se manifiesta de formas muy diferentes y que se traduce asimismo en el ámbito escolar. Esta investigación de carácter descriptivo intenta estimar el estado de la formación que reciben los futuros profesores a través del "Máster Universitario en Formación del Profesorado de Educación Secundaria Obligatoria y Bachillerato, Formación Profesional, Enseñanza de Idiomas y Enseñanzas Artísticas" de la Universidad de Murcia con respecto a la gestión de la diversidad de creencias desde el paradigma de la Educación Intercultural para la escuela laica. A partir de una encuesta, previamente validada por un grupo de expertos, se invitó a expresar su percepción a la totalidad del profesorado con docencia en el curso académico 2013/2014. A pesar de la significativa mortandad de la muestra, los resultados señalan que la formación en estos temas se encuentra en una situación de precariedad. El análisis ha revelado una serie de dificultades y controversias que frente a las necesidades de la escuela suponen, según los datos que se ofrecen, un problema que merece consideración. En conclusión, esta investigación desvela un déficit sensible que, en atención a los argumentos que se esgrimen en la primera parte del presente trabajo en relación a la multiculturalidad y en promoción de una educación más integradora y democrática, precisa de una reflexión transformadora y profunda en un momento en el que se hace especialmente necesaria la educación para la convivencia y la paz.

Palabras clave: Diversidad, Religión/es, Educación Intercultural, Formación del Profesorado, educación, enseñanza universitaria.

\footnotetext{
Abstract:

Our current context is facing a rich outlook in the matter of diversity of beliefs, manifested in many different ways and that is also present at schools. This research tries to analyze, in a descriptive way, the training situation that future teachers are receiving through
} 
the "Máster Universitario en Formación del Profesorado de Educación Secundaria Obligatoria y Bachillerato, Formación Profesional, Enseñanza de Idiomas y Enseñanzas Artísticas" (Master's Degree in Teacher Training for Compulsory Secondary Education and Baccalaureate, Vocational Training, Language Teaching and Artistic Teaching) offered by the University of Murcia regarding the management of diversity of beliefs from the point of view of Intercultural Education for secular education. By means of a survey validated by a group of experts as the starting point, all the members of academic faculty lecturing during the academic year 2013-2014 were invited to express their perceptions. Even though it was scarcely answered, the results point out that this specific kind of training is currently in a precarious situation. The analysis revealed a series of difficulties and controversies that based on the data provided, and in view of the school needs, pose a problem which deserves special consideration. In conclusion, this research shows a remarkable deficit in respect of the arguments mentioned in the first part of the article concerning multiculturalism and promotion of a more unifying and democratic education. This situation requires then a transforming and profound reconsideration, even more in a context in which education for coexistence and peace is primarily necessary.

Keywords: Diversity, Religion, Multicultural Education, Teacher Education, education, university teaching.

\section{Una aproximación a la diversidad}

El desarrollo social de España en las últimas décadas ha ocasionado que nuestra sociedad actual se caracterice por contar con un rico y variado tejido multicultural cuya diversidad conforma, al día de hoy, una especialidad y un rasgo cardinal en el entendimiento de nuestra propia identidad nacional. Esta diversidad se manifiesta y tiene implicaciones en una infinidad de dimensiones, constituyendo una realidad compleja y poliédrica que al día de hoy presenta una gran cantidad de oportunidades para la investigación desde distintas áreas.

Desde una aproximación biológica y/o antropológica el ser humano es único e irrepetible, por tanto diverso, de modo que la diversidad es una condición natural de la mismidad del ser humano. Pero, desde el campo socioeducativo, ésta puede adquirir implicaciones negativas pues una cosa es la diversidad y otra muy distinta los diversos. Hay diversidades que causan ternura, tristeza, compasión pero también hay diversidades que causan temor, miedo y hasta repulsión, especialmente entre aquellos que no se consideran diversos, aunque también en los diversos que se cambiarían por otros pero no pueden (Almeida et al., 2010).

Sin embargo, la percepción derivada de la prensa y la observación cotidiana se aproxima más a la homogeneidad que a la diversidad de creencias. Los datos aportados por el CIS (2015) señalan que un $69.3 \%$ se confiesan cristianos católicos, un $16.0 \%$ no religiosos, un $10.3 \%$ ateos, un $1,9 \%$ creyentes de otras religiones y un $2.1 \%$ no responde, pero la pregunta que formula, ¿cómo se define usted en materia religiosa: católico/a, creyente de otra religión, no creyente o ateo/a? no deja lugar a un análisis más amplio.

Una mayor aproximación a la temática nos permite descubrir que en el grupo mayoritario de católicos, si bien encontramos un importante marco común, la multiplicidad de vocaciones no nos permite hablar de una identidad única. A partir de la segunda mitad del siglo XX, dentro de la estructura general de la Iglesia Católica han ido emergiendo nuevos movimientos eclesiales o movimientos laicos que se caracterizan, según Muñana (2009), por tener un origen carismático y misionero en una sociedad supuestamente secularizada, generalmente vinculados a un fundador y con suficientes especificidades (carisma, doctrina, 
espiritualidad, metodología) como para ser considerados constituyentes de diversidad. Por su extensión en nuestro país, González Ortiz (2004) destaca el Camino Neocatecumenal, el Movimiento Familiar Cristiano, la Renovación Carismática, el movimiento de los Focolares y Comunión y Liberación y, aunque no son propiamente movimientos, añade a la lista la prelatura del Opus Dei y las Comunidades Cristianas de Base. Todas ellas dan lugar a una gran variedad de formas de ser iglesia o, dicho de otro modo, propician la heterogeneidad en un grupo al que generalmente se considera homogéneo.

Sobre la porción no creyente y atea que explica el CIS (2015) los autores del presente trabajo consideran que las opciones de respuesta no creyente y ateo/a pueden generar confusión. Por el contrario, el eurobarómetro desarrollado por la Comisión Europea (2005) explica que en nuestro país el $21 \%$ de los encuestados afirmó creer en "un espíritu o fuerza vital", mientras que el $18 \%$ no cree "en un espíritu, dios o fuerza vital". Así, se reconoce la presencia de un importante grupo que presenta posturas agnósticas pero queda aún sin esclarecer si estas posturas son de carácter fuerte, débil, apático, interesado o más relacionado con el igteísmo. Este análisis sitúa nuestro país seis puntos por debajo de la media europea (27\%) en cuanto a población que se considera agnóstica, y en la media en cuanto a población atea (18\%). Tampoco se encontraron datos significativos en cuanto a la diversidad que puede existir en la adopción del ateísmo.

Partiendo de la dificultad de encontrar estudios cuantitativos sobre las poblaciones que participan de otras religiones resulta valioso el análisis que el Observatorio del Pluralismo Religioso en España (2013) realiza sobre el número y distribución de lugares de culto por confesiones, lo que permite visualizar la diversidad interna de las religiones minoritarias entendiendo que en torno a cada uno de estos lugares existirá una comunidad, más o menos numerosa, que participa de esa creencia. De los 6.125 lugares de culto incluidos en el Directorio de lugares del culto, el $57.49 \%$ son iglesias evangélicas, el $21.36 \%$ son oratorios musulmanes o mezquitas y el $11 \%$ son salones del Reino de los Testigos de Jehová. En menor proporción se encontraron lugares de culto ortodoxos (2.87\%), budistas (2.22\%) y del Movimiento de los Santos de los Últimos Días (1.88\%).

De igual modo, con respecto a la población musulmana, el último estudio demográfico del Observatorio Andalusí (2015) apunta que actualmente los musulmanes representan el 3.9\% de la población. Asimismo se destaca en este informe la singularidad, en un análisis por provincias de la región Murcia por el número de alumnos musulmanes, tan solo por detrás de Madrid y Barcelona. También se subraya en este estudio la diversidad de creencias dentro de este grupo que generalmente es considerado homogéneo. Los "ritos malekí y hanafí (suníes) son los más extendidos en España para la práctica del culto islámico, seguidos en menor medida del chafeí y del hanbalí, también suníes, y del yafarí (chií)" (p.2), además "algunas pequeñas y apreciadas cofradías sufíes" (p.2). Según este informe, en torno al $40 \%$ de la población musulmana es española y el $60 \%$ inmigrante. De esta manera, y considerando asimismo las muchas otras minorías religiosas que pueden también estar ligadas al fenómeno migratorio, la diversidad cultural y religiosa resultante del incremento de población inmigrante se suma a la diversidad propia de la población autóctona generando un verdadero crisol de confesiones que conforman un fenómeno social cuyas implicancias educativas no deberían subestimarse.

Asimismo, diversos estudios de carácter prospectivo señalan importantes curvas de crecimiento en las creencias religiosas tradicionales, desdiciendo los supuestos procesos de secularización que en algunos casos se suponían consustanciales a la modernidad. 
Sirva de ejemplo el informe publicado en 2015 por el Pew Reseach Center que pronostica para 2050 la continuidad predominante del Cristianismo -en torno al 31.4\% de la población mundial-, un aumento de la creencia en el Islam de hasta un $29.7 \%$ y un descenso del $16.4 \%$ al 13.2\% en la porción de ciudadanos que se confiesan ateos, agnósticos o no afiliados. Esta misma institución estima así la diversidad de creencias en nuestro país para mediados de siglo: un $65.2 \%$ de cristianos, un $7.5 \%$ de musulmanes, un $26.5 \%$ de ateos, agnósticos o no afiliados y menos del $0.1 \%$ de otras religiones (Pew Research Center, 2015).

Aunque tomamos con cierta precaución estos datos especulativos, se pone en entredicho que en el caminar hacia sociedades más modernas lo religioso y su expresión representen un fenómeno obsoleto, aislado y minoritario con una clara proyección exclusivamente interior $^{1}$ y con una tendencia encarecidamente menguante. Desde finales del siglo XX las religiones se están enfrentando a un proceso de desprivatización, rechazando el papel marginal e íntimo de las teorías de la modernidad y la secularización y enfatizando la vocación exterior ${ }^{2}$ y su impacto en la esfera pública. El "resultado es que las distintas formas y posibilidades de lo religioso, espiritual y secular genera un abanico, cada vez más amplio, de identidades personales y grupales variadas y frágiles que interactúan dentro del espacio público" (Escribano, 2013, p. 309), generando una ciudadanía critica que interroga a las instituciones a la espera de respuestas significativas y éticas.

\section{Educación y creencias culturales-religiosas}

Este espacio legítimo de desarrollo no siempre es fácil de delimitar. Hace dos décadas Escolano (1997) anunciaba que el docente tendría que tratar, cada vez con mayor frecuencia, con grupos humanos complejos y plurales en los que cohabitarían minorías étnicas, culturales, religiosas y sociales. Para Touriñán (2008), aunque la decisión es clara: "la escuela debe asumir su responsabilidad cívica" (p. 41), la toma de decisiones suele generar controversias pues "hay un límite a la elasticidad de la tolerancia en las sociedades abiertas y pluralistas que nos obliga a definir y a decidir entre dos valores: el valor de la diversidad cultural y el valor de la igualdad de derecho de todos" (p. 40).

Así, la escuela debe arbitrar en multitud de conflictos, de distinta índole, que surgen naturalmente de la convivencia, relacionados especialmente con la discriminación, la xenofobia y otras actitudes negativas que según Rodríguez Hidalgo (2010) se encuentran en la base de muchos casos de bullying o cyberbullying. También debe evitar la asimilación y delimitar los espacios y los canales adecuados para la expresión de las distintas identidades de los alumnos y sus familias junto con el respeto a la laicidad del espacio escolar. Asimismo debe estudiar y decidir sobre la legitimidad de estas demandas, negociarlas y actuar en consecuencia. Esta toma de decisiones implica manipular una serie de variables muy delicadas entre las que figuran derechos fundamentales. Por tanto, afrontar esta tarea supone también asumir el conflicto.

No solo entendemos esta diversidad como un problema que la escuela debe resolver "sino como medio de tener una vida intelectual, afectiva, moral y espiritual más enriquecedora" (ONU, s.f.). Aunque, como advierten Peña-Ruiz y Tejedor (2009), "la escuela no es un lugar cualquiera donde podría aplicarse el mismo régimen de libertad que en la calle, entre adultos [...] los alumnos menores van a la escuela a instruirse y no para actuar como ciudadanos formados" (p. 56).

La educación en otras formas de creencias puede ser favorable en tres dimensiones: en primer lugar para la reafirmación de la propia identidad con respecto a la diversidad; en segundo lugar para comprender al otro como ser humano en fraternidad más allá de los 
La formación del profesorado ante la diversidad de creencias religiosas en el máster de formación...

accidentes externos ${ }^{3}$ tanto para colaborar en alcanzar metas comunes como para sentir compasión y reaccionar ante la injusticia; y, por último, puede disminuir el riesgo de caer en actitudes o dinámicas sectarias, radicales o fanáticas para lo que la adolescencia (como crisis) supone una etapa de riesgo. Para esto, como explica Jiménez Tallón (1997), la clave está en "educar en libertad y para la libertad, diferenciando los compromisos vitales y sociales lícitos de los yugos ideológicos que esclavizan" (p. 126).

Lo cierto es que desde la Educación Intercultural podemos entender el espacio escolar como un contexto de socialización privilegiado en el que el alumno puede ensayar, de forma mediada y protegida el contacto con otras formas de entender la creencia favoreciendo el pensamiento crítico y poliédrico, además del diálogo y el respeto. Pero, a pesar de estas exhortaciones, la respuesta no ha sido la más indicada. Como explica Gervilla Castillo (2013), "para los humanos, las religiones han sido -y continúan siendo- un factor relevante en la vida personal, social y educativa, a pesar del escaso número de trabajos que se publican en las revistas de educación españolas al respecto" (p. 141), lo que demanda la reflexión sobre la praxis docente y sobre la propia formación del profesorado, eje sobre las que se ha puesto la atención en esta investigación.

Dentro del ámbito de la educación, y más específicamente la educación escolar, resulta fundamental que los futuros profesores se formen en la diversidad en cualquiera de sus modalidades. Si realmente queremos educar para la vida, esta finalidad educativa surge como requisito de la sociedad multicultural en la que vivimos, pero también como la esperanza para posibilitar una escuela inclusiva e intercultural centrada en la igualdad y en la conciliación con los otros (aprender a vivir juntos). Difícilmente podemos educar en lo que no tenemos, en lo que desconocemos cognitiva, afectiva y conductualmente, especialmente en cuanto a lo que a valores y a estilo de vida se refiere. La formación de todo profesorado comienza en la universidad. Luego se continúa en el propio contexto profesionalizador y en las posibilidades de educación permanente que se brindan a los estudiantes y en las que libre y voluntariamente participan. En esta ocasión el presente trabajo se limita a identificar en qué medida la diversidad de creencias, entre ellas las religiosas, constituye un objeto educativo en el máster para la Formación de la Universidad de Murcia.

\section{Método}

Desde 1972, con los primeros Certificados de Aptitud Pedagógica, la Universidad de Murcia ha venido siendo un centro de referencia para la formación del profesorado y ha ido evolucionando según se han ido desarrollando las normativas hasta esta nueva fórmula del máster que, durante el curso 2013-2014, ha preparado y titulado a 322 alumnos. Concretamente, el presente trabajo tiene como objetivo conocer la percepción del profesorado del Máster Universitario en Formación del Profesorado de Educación Secundaria Obligatoria y Bachillerato, Formación del Profesorado, Enseñanzas de Idiomas y Enseñanzas Artísticas, en todas las especialidades y sobre la relevancia de la diversidad de creencias como componente en la formación del profesorado. Esta finalidad se articula en los siguientes objetivos específicos:

Averiguar qué importancia otorgan a estos contenidos dentro de la formación inicial y continua del profesorado en educación secundaria.

Inquirir el valor que dan a estos contenidos en relación con el desempeño profesional del profesor de educación secundaria. 
Conocer si estos contenidos sobre diversidad religiosa se abordan durante el desarrollo de sus asignaturas, tanto si aparecen explicitados en la Guía Docente como si no (currículo oculto).

Conocer cómo valoran la posibilidad de incorporar estos contenidos al corpus del máster o a los propios de su asignatura, en el caso de que no se contemplen.

Se trata de un estudio de naturaleza descriptiva cuyo enfoque metodológico se caracteriza por variables alfanuméricas (dimensión cuantitativa). Para realizar este análisis se ha elaborado un cuestionario compuesto de 29 ítems, validado a través de la técnica Delphi con dos circulaciones y dos planteles distintos de expertos (10 expertos), lo que permitió recoger la opinión del profesorado participante en la docencia del máster sobre distintas cuestiones relacionadas con las creencias y su espacio en el contexto escolar, las necesidades del profesorado en relación a estas y el estado de la formación en relación a sus asignaturas. Se ha solicitado a cada uno de los participantes el grado de acuerdo o desacuerdo respecto al ítem mediante una escala de significación numérica. Los ítems se organizaron en función de los objetivos específicos formulados, siendo el objetivo 2 el que aglutina mayor número de ítems (tabla 1).

En lo que respecta a la fiabilidad del instrumento, por consistencia interna se ha utilizado el estadístico Alfa de Cronbach, que arroja un valor de 0.899, lo cual indica que el instrumento en su totalidad está compuesto, realmente, por ítems que miden la percepción docente sobre la relevancia de la diversidad de creencias. A continuación, en la tabla 1 puede comprobarse que el instrumento también presenta una alta fiabilidad por dimensiones.

Tabla 1. Coeficiente de fiabilidad por dimensiones especificando las variables que agrupan.

\begin{tabular}{|l|c|c|}
\hline Dimensión & Variables & Fiabilidad \\
\hline 1. Características sociodemográficas & - & No procede \\
\hline 2. Formación inicial y continua & 7 ítems & 707 \\
\hline 3. Desempeño profesional & 12 ítems \\
\hline 4. Contenidos disciplinares & 6 ítems & 899 \\
\hline 5. Compromiso futuro & 4 ítems & 851 \\
\hline TOTAL & ítems: 29 & 899 \\
\hline
\end{tabular}

Se realizó un muestreo intencional donde el criterio determinante en el número de la muestra estaría condicionado no tanto por la accesibilidad -ya que el cuestionario se envió por correo electrónico a la totalidad de los docentes del máster en el curso académico 2013-2014, concretamente 177 profesores- sino por la disposición de dichos docentes a cumplimentarlo. El cuestionario cumplimentado se depositaba en un buzón de correo situado en la consejería de la Facultad de Educación de la Universidad de Murcia para garantizar el anonimato, o bien se enviaba a través del correo electrónico. Finalmente, tras dos envíos más por parte de los investigadores la muestra quedó definitivamente compuesta por 53 participantes, lo que evidencia una elevada muerte muestral acusada por la sobrecarga de funciones asumidas por el profesorado universitario y por la temática en cuestión.

La muestra real quedó configurada con las siguientes características: 21 profesoras (39.6\%) y 32 profesores (60.4\%), en los siguientes intervalos de edad: menores de 30 años, 3.84\%; entre 31 y 40 años, 21.15\%; entre 41 y 50 años, $40.38 \%$ (el grupo más numeroso); entre 51 y 60 años, 26.92\%, y mayores de 60 años, 7.69\%. Entre ellos se encontraban 1 ingeniero, 14 licenciados y 36 doctores, que forman parte del cuerpo docente de distintas facultades. 
La formación del profesorado ante la diversidad de creencias religiosas en el máster de formación...

Con respecto al tratamiento estadístico de los datos se ha empleado la aplicación informática IBM SPSS 19.0 Statistics. Se realizó un estudio descriptivo (medidas de tendencia central y dispersión) de las dimensiones consideradas en el instrumento.

\section{Resultados}

A continuación se exponen los resultados atendiendo a los objetivos previamente formulados que se corresponden, a su vez, con cada una de las dimensiones del cuestionario.

\section{Formación inicial y continua del profesorado en educación secundaria}

La percepción de los docentes que imparten en el Máster de Formación del Profesorado de Secundaria sobre la importancia de los contenidos de diversidad religiosa en la formación inicial y continua muestran cierta tendencia predominante a escoger el punto neutral situándose, tal y como evidencian las medias de los distintos ítems que componen esta dimensión (Tabla 2), en el valor 3, es decir, ni de acuerdo ni en desacuerdo.

Tabla 2. Porcentajes y estadísticos descriptivos de la importancia que otorgan los docentes del máster a los contenidos sobre diversidad de creencias en la formación inicial y continua del profesorado de secundaria.

\begin{tabular}{|c|c|c|c|c|c|c|c|}
\hline \multirow{2}{*}{ Ítem } & \multicolumn{7}{|c|}{ Frecuencia de respuesta en $\%^{4}$} \\
\hline & 1 & 2 & 3 & 4 & 5 & $\sigma$ & $x$ \\
\hline $\begin{array}{l}\text { 1. Considero que la formación inicial en } \\
\text { materia de diversidad religiosa es importante } \\
\text { para el desempeño profesional del docente } \\
\text { en E.S. }\end{array}$ & 13.2 & 11.3 & 45.4 & 24.5 & 5.6 & 1.06 & 2.98 \\
\hline $\begin{array}{l}\text { 2. Creo que la formación inicial del } \\
\text { profesorado en este ámbito le hace más } \\
\text { sensible y más eficaz a la hora de atender a } \\
\text { la diversidad. }\end{array}$ & 11.3 & 11.3 & 35.8 & 33.9 & 7.5 & 1.09 & 3.15 \\
\hline $\begin{array}{l}\text { 3. Considero que la formación inicial del } \\
\text { profesorado en este ámbito es indispensable } \\
\text { para el desarrollo de un modelo de escuela } \\
\text { más intercultural e inclusivo. }\end{array}$ & 16.9 & 18.9 & 35.8 & 20.7 & 7.5 & 1.17 & 2.83 \\
\hline $\begin{array}{l}\text { 4. Considero innecesaria la formación inicial } \\
\text { en estos aspectos tan específicos, ya que los } \\
\text { mismos podrían tratarse en la formación } \\
\text { continua y la especialización docente. }\end{array}$ & 9.4 & 18.9 & 26.4 & 37.7 & 7.5 & 1.12 & 3.15 \\
\hline $\begin{array}{l}\text { 5. Además de la formación inicial, creo } \\
\text { necesario que el profesor de E.S. reciba } \\
\text { formación continua en este ámbito }\end{array}$ & 30.2 & 26.4 & 28.3 & 15.1 & - & 1.1 & 2.28 \\
\hline $\begin{array}{l}\text { 6. Considero que la formación en este ámbito } \\
\text { es importante para el desempeño de las } \\
\text { funciones propias de la tutoría en E.S. }\end{array}$ & 13.2 & 33.9 & 16.9 & 26.4 & 9.4 & 1.2 & 2.85 \\
\hline $\begin{array}{l}\text { 7. Considero que para desarrollar una } \\
\text { atención escolar más individualizada es } \\
\text { necesaria la formación previa del profesor } \\
\text { en materia de identidad religiosa. }\end{array}$ & 20.7 & 24.5 & 26.4 & 24.5 & 3.77 & 1.18 & 2.66 \\
\hline
\end{tabular}


Un análisis más pormenorizado permite comprobar que, efectivamente, en el ítem 1 que ahonda en la importancia de la formación en diversidad de creencias para el desempeño profesional del docente de secundaria, la opinión queda dividida en tres sectores donde están prácticamente igualadas las adhesiones (30.19\%), las disensiones (24.53\%) y la neutralidad (45.28\%).

Sin embargo, mientras que en las aversiones la mayoría se sitúa rotundamente en desacuerdo, no puede decirse lo mismo de las adhesiones ya que tan solo un $5.7 \%$ de los encuestados afirman estar muy de acuerdo con que la diversidad de creencia es importante para el desempeño profesional en secundaria. Esta diferenciación entre las percepciones rotundas de los docentes adquiere mayor distinción conforme se avanza en los ítems, de modo que se evidencia que casi un tercio de los encuestados se muestra totalmente en desacuerdo respecto a la necesidad del profesorado de secundaria de recibir formación continua en estos contenidos, mientras que nadie se muestra totalmente de acuerdo.

Paradójicamente, casi el $42 \%$ de los docentes del máster considera, mostrándose de acuerdo y muy de acuerdo, que recibir formación inicial sobre diversidad de creencias contribuye a mostrar mayor sensibilidad y eficacia en la atención a la diversidad del alumnado de secundaria. Sin embargo, el 45\% cree innecesaria la formación inicial en estos aspectos tan específicos relegando los mismos a la formación continua y/o especializada. Para concluir, señalar que si tenemos en cuenta que el ítem 4 es negativo, al transformarlo a positivo tan solo en dos de los siete ítems la adhesión a favor de una formación inicial y continua en el profesorado de secundaria adquiere mayores porcentajes que la repulsión, donde los porcentajes oscilan entre el $22.6 \%$ alcanzado en el ítem 2 y el $56.5 \%$ del ítem 5 .

\section{Valor que los docentes conceden a estos contenidos en relación con el desempeño profesional del profesor de educación secundaria}

En esta ocasión se rompe con la tendencia neutral expuesta anteriormente, pues solo 2 de los 12 ítems que conforman esta dimensión recogen el porcentaje mayoritario de respuestas (tabla 3). Concretamente, casi el 51\% de los docentes del máster no se muestra ni de acuerdo ni en desacuerdo con que la responsabilidad de la atención a la diversidad y la prevención y resolución de conflictos que pueden derivarse de la diversidad de creencias sea del profesor. De igual forma el $32.7 \%$ se muestra indiferente ante la consideración de que la mayoría de los centros de nuestro contexto presentan un panorama homogéneo. 
Tabla 3. Porcentajes y estadísticos descriptivos de la valoración que los docentes conceden a estos contenidos en relación con el desempeño profesional del profesor de educación secundaria.

\begin{tabular}{|c|c|c|c|c|c|c|c|}
\hline \multirow{2}{*}{ Ítem } & \multicolumn{7}{|c|}{ Frecuencia de respuesta en $\%^{5}$} \\
\hline & 1 & 2 & 3 & 4 & 5 & $\sigma$ & $x$ \\
\hline $\begin{array}{l}\text { 8. Entiendo que el desconocimiento de la } \\
\text { diversidad religiosa puede llevar a ignorar } \\
\text { situaciones de conflicto, discriminación o } \\
\text { marginación en los centros. }\end{array}$ & 7.5 & 15.1 & 15.1 & 43.4 & 18.9 & 1.2 & 3.5 \\
\hline $\begin{array}{l}\text { 9. Creo que un profesor con escasos } \\
\text { conocimientos sobre la materia puede } \\
\text { encontrar dificultades para gestionar la } \\
\text { diversidad religiosa en su aula. }\end{array}$ & 7.5 & 24.5 & 24.5 & 30.2 & 13.2 & 1.2 & 3.2 \\
\hline $\begin{array}{l}\text { 10. Reconozco que el conocimiento de la } \\
\text { creencia del alumno ayuda a tener una } \\
\text { comprensión más profunda de éste y de su } \\
\text { entorno. }\end{array}$ & 7.7 & 9.6 & 26.9 & 44.2 & 11.5 & 1.1 & 3.4 \\
\hline $\begin{array}{l}\text { 11. Considero que la expresión de la } \\
\text { identidad religiosa del alumno corresponde } \\
\text { exclusivamente al ámbito privadoy, por tanto, } \\
\text { no tiene razón de ser en el contexto escolar. }\end{array}$ & 5.6 & 15.1 & 22.6 & 16.9 & 39.6 & 1.3 & 3.7 \\
\hline $\begin{array}{l}\text { 12. Entiendo que la atención a la diversidad y } \\
\text { la prevención y resolución de conflictos que } \\
\text { pueden derivar de ésta son responsabilidad } \\
\text { del profesor de E.S. }\end{array}$ & 9.4 & 18.9 & 50.9 & 15.1 & 5.6 & 0.97 & 2.89 \\
\hline $\begin{array}{l}\text { 13. Considero que el profesor de E.S. puede } \\
\text { llevar a cabo una valiosa aportación pedagógica } \\
\text { favoreciendo el diálogo interreligioso en su } \\
\text { aula. }\end{array}$ & 11.3 & 15.1 & 24.5 & 33.9 & 15.1 & 1.22 & 3.26 \\
\hline $\begin{array}{l}\text { 14. Considero que la ignorancia de la diversidad } \\
\text { religiosa del alumnado puede desproteger } \\
\text { algunos derechos fundamentales del } \\
\text { alumnado. }\end{array}$ & 13.2 & 11.3 & 30.2 & 32.1 & 13.2 & 1.21 & 3.21 \\
\hline $\begin{array}{l}\text { 15. Creo que la diversidad religiosa puede tener } \\
\text { implicaciones en el diseño de la programación } \\
\text { docente en E.S. }\end{array}$ & 33.9 & 20.8 & 15.1 & 22.6 & 7.5 & 1.37 & 2.49 \\
\hline $\begin{array}{l}\text { 16. Entiendo que el desconocimiento de la } \\
\text { creencia religiosa o no creencia de la familia } \\
\text { del alumno puede ser un obstáculo para la } \\
\text { comunicación y la colaboración. }\end{array}$ & 26.9 & 15.4 & 23.1 & 28.8 & 5.7 & 1.30 & 2.71 \\
\hline $\begin{array}{l}\text { 17. Estimo que la mayoría de los centros de } \\
\text { nuestro contexto presentan un panorama } \\
\text { homogéneo en este sentido, sin apenas } \\
\text { diversidad religiosa entre el alumnado. }\end{array}$ & 13.5 & 28.8 & 32.7 & 19.2 & 5.76 & 1.10 & 2.75 \\
\hline $\begin{array}{l}\text { 18. Creo que actualmente la identidad religiosa } \\
\text { ha dejado de ser un componente fundamental } \\
\text { en la identidad personal. }\end{array}$ & 9.6 & 28.8 & 26.9 & 23.1 & 11.5 & 1.18 & 2.98 \\
\hline $\begin{array}{l}\text { 19. Considero que estos contenidos son } \\
\text { necesarios para el desempeño propio de la } \\
\text { acción tutorial. }\end{array}$ & 22.6 & 16.9 & 3.9 & 20.7 & 5.6 & 1.20 & 2.70 \\
\hline
\end{tabular}


Desde el análisis de las medias obtenidas en esta dimensión debemos señalar de este apartado la importancia del ítem $n^{\circ} 11$, que indaga sobre la creencia en torno a los espacios de legitimidad, la privacidad o el sentido de la expresión religiosa particular del alumno en la escuela. Puede verse que la suma de las adscripciones (que expresan lo impropio de la expresión de la creencia) alcanza el 56.6\%, siendo además la forma más contundente la mayoritaria (39.62\%), alcanzando la media más elevada 3.70 de todas las registradas. Sin embargo, atendiendo al objeto de estudio de la investigación, cualquier intento de relegar la diversidad religiosa al ámbito privado de la familia o de lo estrictamente personal sería contraproducente para la integración curricular de los contenidos sobre diversidad religiosa. Por lo tanto se trata de un ítem negativo, al igual que el ítem 18, donde se afirma que los aspectos religiosos no son relevantes en la identidad personal.

Por una parte, otra media que sobrepasa el valor neutral con un 3.42 ha sido registrada en el ítem que permite reconocer que el conocimiento de la creencia del alumno ayuda a tener una comprensión más profunda de éste y de su entorno, donde el 55.7\% de los docentes se muestran de acuerdo o muy de acuerdo. En contraposición, el valor medio más bajo, correspondiente al 2.49, se ha obtenido en el ítem que nos permite medir si los docentes consideran que la diversidad religiosa tiene implicaciones en el diseño de la programación docente en educación secundaria pues el 54\% se ha mostrado en desacuerdo o muy en desacuerdo con dicha afirmación.

Por otra parte, los datos relativos al ítem sobre la percepción de la homogeneidadheterogeneidad de esta diversidad en los centros de nuestro contexto escolar muestran que, de forma mayoritaria, se desconoce (32.69\%) o no se reconoce (42.31\%) la presencia de esta diversidad. Estos resultados, que contrastan abiertamente con lo expresado en la introducción, son analizados con más detalle en el apartado Discusión y conclusiones. Interrogados sobre la actualidad de la relevancia de las religiones o de la creencia religiosa en la configuración de la identidad personal (ítem 17, expresado de forma negativa) considerando la identidad como el eje de la educación centrada en la persona, la mayoría de los encuestados (38.46\%) mostró desacuerdo con la afirmación pues considera a la creencia como un factor vigente y de relevancia en la configuración de la personalidad, frente al 34.62\% que rechazó esta idea.

En la búsqueda de una tendencia en las respuestas de los docentes que nos permitiera comprobar el valor que conceden a la diversidad religiosa en el desempeño profesional hemos procedido a agrupar los valores de adhesión y de aversión y hemos podido comprobar que, tal y como muestran los respuestas al ítem 19, no se reconoce que estos contenidos sean necesarios para la acción tutorial, sumando la opción neutral a la mayoría de respuestas (33.96\%), seguido del rechazo rotundo (22.64\%) más el 16.98\% que expresó desacuerdo. Asimismo, y tal como se ha evidenciado, no se estima mayoritariamente que el desconocimiento de la creencia pueda ser un obstáculo para la comunicación entre la familia y la escuela (16) ni para las labores propias de la tutoría (6).

\section{La diversidad de creencias como contenido disciplinar}

Con estos ítems tratamos de conocer la percepción del profesorado sobre el estado de este tipo de formación: en qué medida la diversidad de creencias supone un componente dentro de la formación que se ofrece. Los resultados se presentan en la siguiente tabla nº 4 . 
Tabla 4. Porcentajes y estadísticos descriptivos en relación a la estimación que hace el profesorado de la presencia de estos contenidos en la formación ofrecida a través del máster.

\begin{tabular}{|c|c|c|c|c|c|c|c|}
\hline \multirow{2}{*}{ Ítem } & \multicolumn{7}{|c|}{ Frecuencia de respuesta en $\%^{6}$} \\
\hline & 1 & 2 & 3 & 4 & 5 & $\sigma$ & $\mathbf{x}$ \\
\hline $\begin{array}{l}\text { 20. La guía docente de la asignatura que } \\
\text { imparto incluye contenidos relacionados con } \\
\text { este tema. }\end{array}$ & 58.5 & 18.9 & 15.1 & 3.7 & 3.7 & 1.09 & 1.75 \\
\hline $\begin{array}{l}\text { 21. Aunque no estén recogidos en la guía, estos } \\
\text { contenidos forman parte del corpus teórico de } \\
\text { mi asignatura. }\end{array}$ & 49.1 & 20.7 & 16.9 & 5.6 & 7.5 & 1.26 & 2.02 \\
\hline $\begin{array}{l}\text { 22. Durante el curso he planificado y } \\
\text { desarrollado actividades que han tratado de } \\
\text { alguna manera esta temática. }\end{array}$ & 63.4 & 13.4 & 13.4 & 5.76 & 3.84 & 1.14 & 1.73 \\
\hline $\begin{array}{l}\text { 23. Durante las clases los alumnos han } \\
\text { demandado o expresado interés por estas } \\
\text { cuestiones. }\end{array}$ & 73.1 & 9.6 & 11.5 & 1.9 & 3.8 & 1.04 & 1.54 \\
\hline $\begin{array}{l}\text { 24. Durante el desarrollo de la asignatura esta } \\
\text { temática ha surgido de forma espontánea (a } \\
\text { través de un comentario, aportación, etc.) }\end{array}$ & 61.5 & 15.4 & 11.5 & 9.6 & 1.9 & 1.12 & 1.75 \\
\hline $\begin{array}{l}\text { 25. Estos contenidos son objeto de evaluación } \\
\text { en mi asignatura. }\end{array}$ & 86.5 & 1.9 & 7.7 & 1.9 & 1.9 & 0.85 & 1.31 \\
\hline
\end{tabular}

\section{Posibilidad de incorporar estos contenidos al corpus del máster}

Para concluir, los últimos ítems del cuestionario estaban orientados a conocer la valoración que el profesorado hace de la posibilidad de incorporar estos contenidos relacionados con la diversidad de creencias en el corpus propio de conocimientos. Los resultados se ofrecen en la siguiente tabla $n^{\circ} 5$.

Tabla 5. Porcentajes y estadísticos descriptivos sobre la valoración que hace el profesorado en relación a la incorporación de estos contenidos al corpus del máster.

\begin{tabular}{|c|c|c|c|c|c|c|c|}
\hline \multirow[b]{2}{*}{ Ítem } & \multicolumn{7}{|c|}{ Frecuencia de respuesta en $\%^{7}$} \\
\hline & 1 & 2 & 3 & 4 & 5 & $\sigma$ & $x$ \\
\hline $\begin{array}{l}\text { 26. En mi asignatura no se trabaja esta cuestión } \\
\text { pero podría incluirse en la guía en algún tema } \\
\text { relacionado, ya que guarda relación con los } \\
\text { contenidos propios de la materia. }\end{array}$ & 55.8 & 19.2 & 17.3 & 5.8 & 1.9 & 1.054 & 1.79 \\
\hline $\begin{array}{l}\text { 27. Estos contenidos no tienen relación con los } \\
\text { propios de mi asignatura pero valoro de forma } \\
\text { positiva que se impartan en otras asignaturas } \\
\text { del máster más afines a los mismos. }\end{array}$ & 17.3 & 11.5 & 40.4 & 17.3 & 13.5 & 1.24 & 2.98 \\
\hline $\begin{array}{l}\text { 28. Creo que es necesario una asignatura } \\
\text { específica que aborde esta temática, en } \\
\text { relación a la Atención a la Diversidad Cultural } \\
\text { y la Educación Intercultural }\end{array}$ & 30.8 & 5.7 & 38.5 & 19.2 & 5.7 & 1.26 & 2.63 \\
\hline
\end{tabular}


Finalmente, el ítem 29 recoge la percepción en relación a la segunda intención fundamental de la investigación: la necesidad de incluir o ampliar la formación del profesorado para la atención de esta diversidad. Este apartado ("29. Creo que es necesario desarrollar con más profundidad o incorporar estas cuestiones a la formación inicial del profesorado de educación secundaria") sumó un mayor número de disensiones (43.14\%; la mitad de ellas de la forma más contundente) frente al $27.45 \%$ que sí expresó adhesión al enunciado (aunque solo el 3.92\% de la forma más rotunda). Destaca nuevamente la preferencia por la opción neutral (generalizada en este apartado), con el $29.41 \%$ de las respuestas.

\section{Discusión y conclusiones}

Por una parte queremos señalar la significativa predilección por la opción neutral evidenciada en 10 de los 29 ítems, acaparando más de una cuarta parte (25.24\%) del total de las respuestas. Por esto constituye un rasgo importante en la interpretación de los resultados la falta de definición y de posicionamiento como consecuencia natural de la falta de reflexión, de interés o de estima por el tema. Otra de las causas se deriva de la propia estructura del máster, que condensa una gran cantidad de contenidos en unos créditos muy limitados y no permite acoger otros contenidos que no se consideren indispensables. Por otra parte puede comprenderse esta opción por la neutralidad como una forma de evitar la controversia. Como explicaba Stenhouse (1975), la neutralidad no es solo un rasgo característico en la cultura del profesorado sino una estrategia de actuación frente a cuestiones incómodas. Por tanto estimamos que estas abstenciones acaban por resolverse en la práctica con el descarte de sus contenidos: en primer lugar por lo limitado del espacio curricular sumado al esfuerzo hercúleo que parece suponer la inclusión de novedades, y en segundo lugar, para evitar la polémica que se considera inherente al desarrollo de sus contenidos. En este caso estaríamos hablando de sentencias de neutralidad pasiva, según el lenguaje de Trilla (1995).

Era difícil de prever que, en una población formada esencialmente por investigadores y expertos, casi el 70\% de ellos declinaría la invitación a participar. Aunque es aventurado estimar las razones de tal negativa, pueden especularse las siguientes: el exceso de trabajo de los docentes en las fechas en las que se realizó la investigación (cercanas al fin del curso) y la reticencia a pronunciarse, aun en el anonimato, sobre asuntos relacionados con la creencia o la religión.

Además de la preferencia por la abstención o la neutralidad encontramos en la interpretación de la percepción docente cierta controversia. Por un lado, se considera que algunos aspectos relacionados con la diversidad de creencia pueden tener implicaciones en la escuela pues el profesor de educación secundaria puede llevar a cabo una valiosa aportación pedagógica que favorezca el diálogo interreligioso en el aula. De este modo el conocimiento sobre las creencias del alumno ayuda a tener una comprensión más profunda de éste y de su entorno. La formación del docente al respecto le hace más sensible y eficaz con respecto a la atención a la diversidad, así como el desconocimiento de la diversidad religiosa puede llevar a ignorar situaciones de conflicto y discriminación, encontrar dificultades para gestionar la diversidad y desproteger algunos derechos fundamentales del alumnado. 
Sin embargo, por otro lado no se estima conveniente la incorporación de estos contenidos a la formación inicial del profesorado de educación secundaria (ítem 29). No se valoró simétricamente que los contenidos relacionados con la diversidad de creencias deban incluirse en las asignaturas del máster afines a los mismos (ítem 27). Aún menos apoyo recibió la alternativa de incluir una asignatura específica donde se aborde esta temática en relación a la Atención a la Diversidad Cultural y a la Educación Intercultural (ítem 28). A la luz de estos resultados podríamos declarar que aunque en cierta medida se reconocen como sustanciales algunos aspectos relacionados con la diversidad de creencia y sus implicaciones en la escuela, se desestima de forma mayoritaria la necesidad de incorporar estos contenidos a la formación inicial del profesorado. En este sentido quizá podríamos entender que se desaprueba la incorporación de estos contenidos en la formación inicial del profesorado porque se considera preferible su estudio en la formación continua y la especialización docente (ítem 4).

Es significativo además que, en contradicción con los datos aportados en la introducción, al preguntar sobre si los centros presentan un panorama homogéneo o heterogéneo con respecto a esta diversidad (ítem 17) casi un tercio (32.69\%) optó por la opción neutral y el $25 \%$ no reconoció la presencia de esta diversidad en nuestros contextos escolares, frente al $42.31 \%$ que sí lo hizo. Por tanto, y como una de las causas que podrían explicar estos resultados, podríamos decir que mayoritariamente se desconoce o se rechaza la idea de que la diversidad de creencias conforma una realidad a considerar en nuestro contexto educativo.

También es subrayable, y quizá otra clave para entender el contrasentido, que en la percepción docente no existe una firme convicción de que la atención a este tipo de diversidad -incluyendo la prevención y la resolución de los conflictos que se derivan de ésta- conforme una responsabilidad sustancial del profesorado en educación secundaria (ítem 12). A este respecto el $50.94 \%$ optó por la respuesta neutral, el $28.3 \%$ negó que fuera competencia del docente, mientras que el $20.75 \%$ sí reconoció el compromiso. De la misma manera, tampoco se consideró mayoritariamente que estos contenidos fueran necesarios para el desempeño característico de la tutoría o la acción tutorial (ítem 6 y 29), no obstante se reconozca (aunque débilmente) que el desconocimiento en materia de diversidad de creencias puede ocasionar dificultades en la gestión de la diversidad o puede llevar a ignorar situaciones de conflicto, discriminación y marginación (ítems 8 y 9 ) ocasionando incluso perjuicio en los derechos fundamentales del alumno (ítem 14), asuntos graves como explicábamos en la introducción que a nuestro juicio sí atañen directamente a la responsabilidad docente y a las funciones propias del tutor en educación secundaria.

Según nuestra experiencia hay alumnos y familias que están necesitando, de forma apremiante, una forma distinta de escuela que les permita disfrutar en plenitud de la escolaridad, sin discriminación y en igualdad de oportunidades. Para esto es indispensable un nuevo modelo de profesor. Defendemos que la escuela y los docentes que participan en ella no pueden cerrar los ojos ni mantenerse neutrales (en el sentido pasivo) frente al conflicto o la injusticia que pueden surgir de la convivencia de los distintos, incluyendo las distintas formas de creencia. Por el contrario, deben ser activos en su administración: la buena gestión de la diversidad, desde la perspectiva de la educación intercultural, no solo ofrece abundantes ventajas educativas sino que supone un aumento significativo y dignificante en la calidad de la experiencia escolar de los alumnos y sus familias, que en demasiadas ocasiones se encuentran vulnerables, sufriendo situaciones de discriminación o en grave riesgo de marginación, fracaso o abandono escolar prematuro. 
Entendemos que existe una amplia diversidad de diversidades: cultural, de creencias, socioeconómica, familiar, funcional, sexual, etc., teniendo todas ellas, cada una en sus formas, distintas implicaciones que caracterizan y definen los contextos y las situaciones que las que el docente debe desarrollar su actividad profesional. Más aún, es el interés y la preocupación por estas particularidades lo que define la educación centrada en la persona inclinándose por un modelo más justo e integrador inspirado en la ética levinasiana, que reivindica la responsabilidad de ofrecer una respuesta educativa al otro y desde el otro como epicentro. Así, defendemos la conveniencia de abrir un proceso de reflexión amplio y colegiado para conocer si se está teniendo en consideración este gran abanico de diversidades o si se está manteniendo una conceptualización estrecha y tasada.

En conclusión, lamentamos el silencio que existe en el plano académico con respecto a la diversidad de creencias como componente de la diversidad en los centros escolares y la escasa formación que parece recibir el profesorado. Así, exhortamos a los académicos, profesionales y responsables de ofrecer esta formación a abrir un proceso de reflexión amplio, valiente y profesional que se nutra de distintas fuentes y que permita incorporar nuevas visiones sobre la diversidad y su gestión, para preparar y sensibilizar de una forma más integral a sus agentes contribuyendo de esta manera a mejorar la escuela y la calidad de la experiencia educativa que ofrece, con especial inclinación hacia los alumnos que se encuentran más vulnerables.

\section{Referencias bibliográficas}

Almeida, M.E., Angelino, M.A., Kipen, E., Lipschitz, A., Marmet, M., Rosato, A. y Zutión, B. (2010). Nuevas retóricas para viejas prácticas: repensando la idea de diversidad y su uso en la comprensión y el abordaje de la discapacidad. Política y sociedad, 47 (1) 27-44.

CIS (2015) Barómetro de enero 2015. Recuperado de: http://www.cis.es/cis/export/sites/ default/-Archivos/Marginales/3040_3059/3050/es3050mar.pdf

Comisión Europea (2005). Eurobarometer. Social values, science and tecnology. Recuperado de: http://ec.europa.eu/public_opinion/archives/ebs/ebs_225 report_en.pdf

Escolano, A. (1997). El profesor del futuro. Entre la tradición y los nuevos escenarios. Revista interuniversitaria de formación del profesorado, 29, 111-115.

Escribano-Cárcel, M. (2013). Teología feminista como instancia crítica de las religiones en el espacio público. La propuesta de Schüssler Fiorenza. Contrastes, Revista internacional de filosofía, 18, 305-320.

Gervilla Castillo, E. (2013). Laicidad, convivencia ciudadana y educación. Revista de educación, 306, 414-435.

González Ortiz, J.J. (2004). Transmisión de valores religiosos en la familia. Murcia: Espigas.

Jiménez Tallón, M.A. (1997). Factores de predisposición y prevención del fenómeno sectario. Carthaginesia. 8, 115-128.

Muñana González, M. (2009). Los nuevos movimientos eclesiales en comunión para la misión. Recuperado de: http://www2.omp.es/OMP/documentos/estudios/spm2009manuelgonza lezmu\%C3\%B1ana.pdf 
La formación del profesorado ante la diversidad de creencias religiosas en el máster de formación...

Observatorio Andalusí (2015). Estudio demográfico de la población musulmana: explotación estadística del censo de ciudadanos musulmanes en España referidos a fecha 32/12/2014. Recuperado de: http://ucide.org/sites/default/files/revistas/estademograf14.pdf

ONU (s.f.). Día mundial de la Diversidad Cultural para el Diálogo y el Desarrollo. Recuperado de: http://www.un.org/es/events/culturaldiversityday/background.shtml

Peña-Ruiz, H. y Tejedor de la Iglesia, C. (2009). Antología laica: 66 textos comentados para comprender el laicismo. Salamanca: Ediciones de la Universidad de Salamanca.

Pew Research Center (2015). The future of word religion: population growth projections: 2010-2050. Recuperado de: http://www.pewforum.org/files/2015/03/PF_15.04.02 ProjectionsFullReport.pdf

Rodríguez Hidalgo, A.J. (2010). Violencia escolar en sociedades pluriculturales: bullying y victimización entre escolares de carácter étnico-cultural. Córdoba: Servicio de publicaciones de la Universidad de Córdoba.

Stenhouse, L. (1975). Neutrality as a criterion in teaching: the work of the Humanities Curriculum Project. En M.J. Taylor (Ed.), Progress and problems in moral education (pp.112113). Windsor: NFER Pub. Company Ltd.

Touriñan López, J.M. (2008). Educación en valores, Educación intercultural y formación para la convivencia pacífica. La Coruña: Netlibro

Trilla Bernet, J. (1995). Educación y valores controvertidos. Elementos para un planteamiento normativo sobre la neutralidad en las instituciones educativas. Revista Iberoamericana de Educación 7, 93-120.

\section{(Endnotes)}

1 Esta proyección interior y exterior de las creencias, entendida como carácter social, ha sido un objeto intenso de estudio por parte de diferentes sociólogos y filósofos de las religiones desde hace décadas. Entre ellos quizá destaquen, como referentes, Durkheim y Malinowsky.

2 Ídem.

${ }^{3}$ Conceptos extraídos del popular discurso I've Been to the Mountaintop de M. Luther King, pronunciado el 3 de abril de 1968 con respecto a la figura del prójimo y el altruismo.

${ }^{4}$ Esta escala de 1 a 5 se corresponde con la escala de significación numérica aplicada en el cuestionario con la siguiente relación: 1. Totalmente en desacuerdo, 2. En desacuerdo, 3. Ni en acuerdo ni en desacuerdo, 4. De acuerdo, 5. Totalmente de acuerdo.

${ }^{5}$ Esta escala de 1 a 5 se corresponde con la escala de significación numérica aplicada en el cuestionario con la siguiente relación: 1. Totalmente en desacuerdo, 2. En desacuerdo, ${ }^{3}$. Ni en acuerdo ni en desacuerdo, 4. De acuerdo, 5. Totalmente de acuerdo.

${ }^{6}$ Esta escala de 1 a 5 se corresponde con la escala de significación numérica aplicada en el cuestionario con la siguiente relación: 1. Totalmente en desacuerdo, 2. En desacuerdo, 3. Ni en acuerdo ni en desacuerdo, 4. De acuerdo, 5. Totalmente de acuerdo.

7 Esta escala de 1 a 5 se corresponde con la escala de significación numérica aplicada en el cuestionario con la siguiente relación: 1. Totalmente en desacuerdo, 2. En desacuerdo, 3. Ni en acuerdo ni en desacuerdo, 4. De acuerdo, 5. Totalmente de acuerdo. 